\title{
VALORACIÓN DE LA CULTURA CIENTÍFICA MEDIANTE ENTREVISTAS. ABORDAJE EN GRUPOS DIFERENCIADOS
}

\author{
Marianella Maxera Abella ${ }^{1}$ y Lucía Álvarez Blanco² \\ Universidad de Oviedo - España - ${ }^{1}$ maxera@adinet.com.uy; ${ }^{2}$ alvarezblucia@uniovi.es
}

\begin{abstract}
Resumen. La cultura científica de personas adultas es una preocupación recurrente desde hace décadas, abordándose su medición desde estrategias cuantitativas, recurso privilegiado sin resultados contundentes; Objetivos: Este trabajo explora nuevas estrategias cualitativas para la valoración de la cultura científica de los ciudadanos más allá de la enseñanza formal, complementando así los trabajos cuantitativos fundamentados en cuestionarios, procedimiento hegemónico hasta el momento a escala mundial; Métodos: La entrevista estructurada realizada con tres grupos homogéneos de personas adultas, diferenciados atendiendo a su actividad laboral o formación postobligatoria alejadas de la ciencia, se dividió en dos bloques de preguntas abiertas, apoyándose el segundo en la lectura por parte del participante de una propaganda publicitaria. Resultados: Se advierten: a) una fuerte vinculación entre la actitud emocional de las personas y la metodología docente en sus inicios escolares; b) una hegemónica visión tradicional de la ciencia y; c) una marcada diferencia en aspectos cognitivos entre quienes cursaron niveles más altos de la enseñanza Secundaria, referidos al pensamiento formal y al uso de modelos. Conclusiones: Esta estrategia de valoración de la cultura científica es acorde con las implementadas desde perspectivas cuantitativas si bien aporta nuevas dimensiones, destacándose la emocional que desvela la incidencia de la escuela en la cultura científica de las personas.
\end{abstract}

Palabras clave: Cultura Científica; Enseñanza de las Ciencias; Ciudadano Científicamente Culto; Entrevista Estructurada.

\section{USING IN-DEPTH INTERVIEWS FOR EXPLORING SCIENTIFIC CULTURE AMONG DIFFERENT GROUPS OF PEOPLE}

\begin{abstract}
Adults' scientific culture has been a common concern for some decades. Its measurement through quantitative strategies has become the privileged resource; however, strong results still being a remaining issue; Purpose: The following work aims to explore new qualitative strategies to measure scientific culture on people beyond formal education, which enhances quantitative instruments such as questionnaires, identified as the hegemonic strategy so far worldwide; Methods: Three homogeneous groups of adults, currently differentiated by their work activity and/or their university education background (both away from science), took part in a in-depth interview divided into two open-question blocks. The second block was based on a participants' reading of an advertising; Results: Results show (a) a strong link between the current attitude of people and their teacher's methodology during their school beginnings, (b) an hegemonic traditional vision of science, (c) a strong difference regarding cognitive aspects among those people who reached the highest levels of secondary education, in relation to formal thinking as well as the use of models. Conclusions This scientific culture measurement strategy converges with those implemented under the quantitative paradigm, yet it provides new dimensions such as the emotional which reveals the incidence of the school in people' scientific culture.
\end{abstract}

Key-words: Scientific Culture; Sciences Teaching; Scientific Literate Citizen, Structured Interview.

\section{INTRODUCCIÓN}

El interés por sopesar la cultura científica de los individuos más allá de los ámbitos escolares aparece con fuerza en el mundo occidental desarrollado hace décadas. Específicamente, aspectos como la necesidad de arrancar la aceptación de la ciudadanía hacia desarrollos científicos-tecnológicos controversiales como los referidos a la energía nuclear, la exploración 
del espacio o, más recientemente, la manipulación genética, originó una serie de propuestas a mediados del siglo pasado para evaluar lo que las personas saben de ciencias, potenciar su acercamiento a ellas y, supuestamente, lograr una actitud positiva hacia la ciencia y/o la tecnología. Si como se menciona, los tópicos explorados han variado a lo largo de la historia, la modalidad en que se evalúa lo que la ciudadanía conoce de ciencia se ha mantenido de forma hegemónica: los cuestionarios de respuesta cerrada. En España, el organismo encargado de implementar estos instrumentos es la Fundación Española para la Ciencia y la Tecnología, que los realiza de forma bianual desde el año 2002 (FECYT, 2017).

Este abordaje cuantitativo, parece no haber desencadenado conclusiones potentes, y los escasos resultados obtenidos durante décadas concluyen que aunque la gente admira la ciencia, declara saber muy poco de ella. Así pues, la pobreza de las conclusiones conduce, por un lado, a una clara insatisfacción incluso de los responsables de diseñar e implementar estos estudios (Cadena-Iñiguez et al., 2017).

Por su parte también, impide una toma de decisiones certeras sobre la consolidación de la cultura científica de las personas en sociedades democráticas que requieren de la participación con una buena base de formación en ciencias (Osorio, 2018).

En este sentido, este documento aborda primeramente el problema de la evaluación de la ciudadanía en cuanto a conocimientos y manejo de la ciencia, así como también la actitud mostrada hacia ella. Para ello se adopta una metodología cualitativa, fundamentada en el paradigma interpretativo (Mena, Troyano, \& Aguilera, 2020).

Si bien la propuesta es el uso conjunto de una selección de instrumentos: entrevista estructurada, grupos de discusión y juego de roles, con los mismos participantes y el mismo investigador, por razones de extensión, el avance presentado en este artículo se vertebra sobre el primero de los instrumentos, la entrevista estructurada, con conocimiento pleno de que los resultados son por ahora preliminares.

En un futuro, los hallazgos globales podrán apoyar los obtenidos desde perspectivas cuantitativas, tomando en cuenta si ambos convergen, divergen o se complementan (Jentoft \& Olsen, 2019; Morgan, 2019). 
Consideramos que el punto de partida en la valoración de la cultura científica de las personas, tanto desde una perspectiva cuantitativa como cualitativa, lo constituye la definición de lo que se entiende por ciudadano científicamente culto.

En este punto se observa consenso respecto a ciertos aspectos, a saber se trataría de una persona que maneja correctamente conceptos científicos y que además está convencida de ellos de manera que esos conceptos tienen impacto en su comportamiento individual y le movilizan emocionalmente. Asimismo, si bien puede o no participar colectivamente, posee las herramientas y la autoconfianza para hacerlo si así este lo desea puesto que estos conceptos científicos forman parte de su sistema cognitivo, encuentran un significado en él y determinan las decisiones futuras. Detrás de estos conceptos existe una forma de razonar característica de las ciencias: propuesta de hipótesis, control de variables, diseño de una experiencia para comprobar si son aquellas correctas y utilización del pensamiento: "si, por lo tanto, entonces".

El ciudadano científicamente culto se interesa por la ciencia: busca, vincula, jerarquiza información científica; también se conmueve con la ciencia y, finalmente entiende que la ciencia es una construcción humana inacabada y cambiante (Costanzo \& Golombek, 2020; Strasser, Baudry, Mahr, Sánchez, \& Tancoigne, 2018). Esta aproximación a una definición nos orienta hacia la selección de dimensiones de estudio.

\subsection{Objetivo}

Implementar una estrategia cualitativa que evalúe la cultura científica de individuos adultos no comprometidos académica ni laboralmente con la ciencia a través del desarrollo de una entrevista estructurada, como parte de un abordaje holístico de naturaleza cualitativa.

\section{METODOLOGÍA}

\subsection{Diseño}

Para describir las cuestiones metodológicas adoptadas en este estudio, en la tabla 1 se presentan las dimensiones insinuadas anteriormente al hilo de la definición de ciudadano científicamente culto.

La ubicación en una pregunta y un bloque es aproximada, pues al ser las preguntas planteadas en forma de guía, algunas dimensiones pueden aparecer en distinto orden. 
Tabla 1: Descripción de las dimensiones, códigos y bloque de la entrevista

\begin{tabular}{|c|c|c|c|}
\hline Dimensión & Descripción de la dimensión & $\begin{array}{c}\text { Código asignado a la } \\
\text { respuesta }\end{array}$ & $\begin{array}{l}\text { Bloque (B) y } \\
\text { Pregunta (P) } \\
\text { correspondient } \\
\text { e en entrevista }\end{array}$ \\
\hline \multirow[t]{2}{*}{ Emocional } & $\begin{array}{l}\text { - Vinculación emocional con los } \\
\text { conceptos científicos y con la situación } \\
\text { de aprendizaje durante sus primeros } \\
\text { pasos con la ciencia }\end{array}$ & Positiva/Negativa & $\mathrm{B} 1 / \mathrm{P} 1$ \\
\hline & - Interés por la ciencia & Existencia/No existencia & B1/P3 \\
\hline Social & $\begin{array}{l}\text { - Confianza y Autoconfianza en la } \\
\text { participación }\end{array}$ & Existencia/No existencia & B1/P4 \\
\hline \multirow[t]{2}{*}{ Epistemológica } & - Visión de ciencia & $\begin{array}{c}\text { Acabada y } \\
\text { estática/inacabada y } \\
\text { cambiante }\end{array}$ & B1/P6 \\
\hline & $\begin{array}{l}\text { - Distinción/valoración de la } \\
\text { ciencia/pseudociencia }\end{array}$ & $\begin{array}{c}\text { Distingue/no distingue } \\
\text { Valora a ambas, valora sólo } \\
\text { una }\end{array}$ & $\begin{array}{l}\text { B1/P5 } \\
\text { B1/P7 }\end{array}$ \\
\hline Cognitiva & $\begin{array}{l}\text { - Manejo de conceptos científicos } \\
\text { - Uso del pensamiento hipotético } \\
\text { deductivo }\end{array}$ & $\begin{array}{l}\text { Correcto/Incorrecto } \\
\text { No se manejan } \\
\text { Se utiliza/No se utiliza }\end{array}$ & B2 \\
\hline
\end{tabular}

Como se puede observar, se parte de un diseño metodológico de naturaleza cualitativa en el que a través de esta propuesta de dimensiones se explorará la percepción y significado que la ciencia posee para la ciudadanía.

\subsection{Participantes}

Como ya se señaló anteriormente, los individuos objeto de estudio no deberían estar comprometidos académica ni laboralmente con la ciencia, motivo por el que se ha adoptado una selección de los participantes no probabilística que impide que los hallazgos obtenidos puedan ser extrapolables a toda la población española. Así pues, los grupos objeto de investigación son tres: 1) actores de teatro; 2) alumnado de la licenciatura de Filosofía de un mismo curso y 3) profesionales forestales en formación. A su vez, cada uno de los grupos está constituido por cuatro sujetos por lo que se trabajará entonces con un total de doce personas de tres perfiles laborales y/o formativos distintos.

Todos los grupos participaron de forma voluntaria si bien mientras los dos primeros (1 y 2 ) recibieron el abono de gastos de desplazamiento y un ágape (dietas), la actividad con el tercer grupo se desarrolló en paralelo a su participación en un curso de capacitación al que asistían.

La franja etaria de los participantes, oscila entre los 20 y 54 años, existiendo varones y mujeres en todas las agrupaciones. 


\subsection{Descripción de los grupos}

\subsubsection{Grupo de actores}

Como grupo a estudiar se ha elegido a personas adultas (dos varones y dos mujeres con edades entre los 26 y 48 años) que forman parte de una compañía de teatro. La actuación escénica en la misma constituye su actividad laboral. Todos poseen estudios de Bachillerato completos excepto una persona que posee un Grado Medio. Esta formación post obligatoria no se vincula con la ciencia pero asegura una buena base de cultura general (algunos poseen una licenciatura y todos muestran un compromiso fuerte con la cultura).

\subsubsection{Grupo de alumnado de la Licenciatura de Filosofía}

Se trata de adultos más jóvenes que los que integran el grupo anterior. También existe una cultura general básica asegurada puesto que todos han realizado el Bachillerato Científico. A pesar de esta formación, los tres varones y la mujer, en la actualidad cursan estudios universitarios de Filosofía no desarrollando ninguna actividad laboral remunerada y limitándose su contacto entre sí a algunas asignaturas que comparten. Sus edades oscilan entre los 20 y 23 años.

\subsubsection{Grupo de profesionales forestales}

Este último grupo posee un perfil netamente práctico. Careciendo de formación post obligatoria, a día de hoy se están especializando en Formación Profesional, realizando cursos teóricos y prácticos (en su mayoría), con salidas de campo encaminadas a la mejora y conservación de montes. Los cuatro participantes (una mujer y tres varones) tienen edades entre 32 y 54 años.

\subsection{Instrumento de recogida de información}

Categorizado dentro de la técnica de encuesta, se ha optado por la entrevista estructurada que permite identificar problemas, sistemas de valores, comportamientos, estados emocionales de las personas a través de un contacto directo con ellas (Olaz, 2008), respondiendo por tanto de manera ideal a los objetivos de este estudio, tal como se adelantó en la introducción y en la definición de cultura científica expuesta. La elección de esta modalidad de entrevista queda justificada por la posibilidad de definir un conjunto de preguntas, que se diseñan de manera lo suficientemente abierta como para que el entrevistado pueda explayarse o aludir a tópicos no definidos previamente (Morgan, 2019). 
Con ello, durante la implementación de este instrumento el investigador asume un rol de guía a través del planteamiento de preguntas, concediéndole al sujeto entrevistado la posibilidad de completar, matizar, corregir o desarrollar sus ideas, favoreciendo con ello su expresión libre y respetando afanosamente la escucha activa (Emans, 2019).

Conforme lo reseñado, se ha configurado, por un lado, un bloque de interrogantes semiabiertos con los que se intenta indagar acerca de la actitud en los primeros acercamientos a la ciencia en la enseñanza obligatoria, así como también su actitud actual hacia la ciencia y la tecnología y su tendencia a participar en cuestiones científicas socialmente vivas. En un segundo momento, y tras solicitar la lectura de una propaganda publicitaria sobre las virtudes de la cáscara de almendras como combustible, se plantean otras cuestiones que intentan sondear los conocimientos y estrategias donde debería aflorar el pensamiento formal.

\subsection{Registro y análisis de la información}

Las entrevistas realizadas individualmente han tenido una duración promedio de 40 minutos, optándose por su registro en audio y posterior transcripción manual. Seguidamente, la información recabada se ha sometido a un análisis de contenido siguiendo las fases e indicaciones de Espín (2002), a saber: 1) Constitución del corpus de análisis; 2) Formulación del objetivo de análisis; 3) Elaboración de indicadores o unidades de análisis: codificación y categorización de las unidades de registro y de contexto y 4) Sistematización e interpretación de los resultados obtenidos.

\section{RESULTADOS}

\subsection{Análisis general del bloque 1: Dimensiones emocional, social y epistemológica}

Pregunta № 1: En la enseñanza obligatoria has cursado materias científicas (Primaria y Secundaria obligatoria las incluyen). ¿Qué sentimientos te evoca el recuerdo de tus clases de ciencias?

De las respuestas surge la existencia de emociones positivas y negativas. Las primeras, referidas por los participantes a través de términos como "gusto", "adoración", "agrado" “diversión, "entretenimiento", "expectativa" o "curiosidad", están asociadas a la metodología empleada por el profesorado y más concretamente a la actividad en clase, concebida esta 
como prácticas o con una gran implicación del alumno que requiere un nivel alto de acción, más allá de la escucha y toma de notas. Así lo expresa una participante:

“...por ejemplo en mi cole teníamos un laboratorio muy bonito, como muy antiguo y eso es como... Salías de clase para ir al laboratorio, eh, utilizabas cosas, entonces claro, eso sí que es verdad que ayuda mucho a que te guste..." (Mujer, 32 años, actriz de teatro, Bachillerato de Letras, bajo interés declarado por la ciencia).

Como reflejan los siguientes comentarios, otros sujetos agregan además como indicador positivo el carácter lúdico de ciertas actividades y la contextualización de los temas enseñados:

“...tuvimos un profesor muy bueno que nos hacía concursos..." (Mujer, 32 años, actriz de teatro, Bachillerato
de Letras, bajo interés declarado por la ciencia).
“...nos llevaban a las fábricas de aquí de Avilés que trabajaban con esos elementos químicos, cómo los
procesaban... Tú lo podías ver..." (Varón, 26 años, actor de teatro, Bachillerato en Ciencias de la Salud,
mediano interés por la ciencia).
“...nos hacía jugar a un bingo para aprendernos la tabla periódica...” (Varón, 20 años, Bachillerato científico,
estudiante de licenciatura de filosofía, alto interés por la ciencia declarado).

Solamente una alumna menciona emociones positivas mediante el término "agrado", vinculándolas a una característica de la ciencia: la posibilidad del uso del lenguaje matemático que le permite reducir, simplificar y sintetizar la multitud de fenómenos del mundo natural.

"...sentimientos muy agradables, buenas sensaciones, me gustaba...el hecho de poder representar tantas cosas, sólo con matemáticas..." (Mujer, 21 años, Bachillerato Científico, estudiante de la Licenciatura de Filosofía, interés medio declarado por la ciencia).

Por su parte, la alusión a emociones negativas se concreta con términos como "odio", "sufrimiento", "hostilidad", "frustración" o "arrepentimiento", y se asocian con la metodología del profesor, específicamente con el recuerdo de aprendizajes memorísticos, por repetición, descontextualizados y sin un significado o utilidad. Este rechazo se advierte en comentarios como los que siguen:

\footnotetext{
"...daban las materias de una manera como muy, muy, muy estricta. Muy: "tienes que estudiarte esto para luego volcarlo en un examen..." (Varón, 26 años, actor de teatro, Bachillerato en Ciencias de la Salud, mediano interés por la ciencia).

"...me acuerdo de lo que yo tenía sufrido por.., por... por lo de los elementos...". "...me resultaba tan hostil, tan hostil, tan hostil, eh... Cada vez que empezábamos con el calcio no sé qué.., el nitrógeno y tal.., todos los símbolos y tal..." (Varón, 43 años, director de teatro, Bachillerato en Letras, Licenciado en Geografía e Historia, interés medio declarado por la ciencia).
}

Quienes se manifiestan de esta manera, mencionan reiteradamente el tema de la "tabla periódica" como uno de los obstáculos vinculados al imperativo de tener que recordar los símbolos de los elementos, sus números atómicos, etc., así como también el de "los vectores", otro concepto abstracto que, sobre todo al principio del tema, eran trabajados desconociendo la razón de ser memorizados. También se alude a emociones negativas referidas a la 
formalización matemática: el pasaje del fenómeno estudiado, fácilmente reconocido de la vida diaria o de su demostración práctica en el aula, a la fórmula en la pizarra es visto como un paso insalvable para algunos. Así lo expresa uno de los participantes:

"...lo que nunca entendía yo, que me gusta más ahora de mayor que en aquella época es, cuando se introducía lo específicamente matemático porque yo en matemáticas era siempre mi..., mi..., mi bestia negra..." "...me repateaba muchísimo y era lo que menos prestaba atención..." (Varón, 43 años, director de teatro, Bachillerato en Letras, Licenciado en Geografía e Historia, interés medio declarado por la ciencia).

Pregunta No 2: En los últimos tiempos han acontecido una serie de reivindicaciones en Asturias a través de movimientos sociales que se organizan en Plataformas. ¿Has participado de alguna manera en alguna de ellas?

A través de esta pregunta se trataba de desvelar el grado de implicación de los participantes en asuntos de tipo social que se tradujeran en una participación activa y concreta en asuntos de su vida y de su comunidad. La intención última es contrastar con la siguiente pregunta, en la que la participación se debería dar en un problema de tipo tecno/científico. De los doce participantes solamente dos se muestran activos: uno a través de las redes sociales y otro a nivel sindical. La amplia mayoría alude al descreimiento en la utilidad de su implicación. A este respecto una informante señala que:

"No... a veces me da la impresión de que soy demasiado descreída con que las cosas pueden funcionar, entonces sí que es cierto que cuando hay una manifestación o hay algo, sí puedo, hago acto de presencia pero luego a la hora realmente de estar en un sitio y estar trabajando allí no lo hago..." o bien "...desconfío de las personas más bien. A veces me da más esa impresión..." (Mujer, 32 años, actriz de teatro, Bachillerato de Letras, bajo interés declarado por la ciencia).

Pregunta No 3: En las propuestas electorales de los partidos políticos, ¿qué áreas son las que consideras más relevantes? A la hora de votar, ¿en qué cuestiones te fijas? En aspectos económicos, sociales, culturales, éticos, religiosos, científicos... ¿Qué es lo que más priorizas?

Aunque se señalan tópicos como la educación o la ciencia, de las respuestas se concluye un reparto mayoritario entre las esferas social y económica. No obstante, algunos responden desde un marcado interés individual:

"Principalmente en lo social. Yo, yo... Yo analizo lo que tengo yo en mi vida ¿no?... Ya no tanto a nivel estatal,... sino que yo me voy un poco en qué me va a beneficiar ese partido político o ese voto que yo voy a dar a mi entorno local..." (Varón, 26 años, actor de teatro, Bachillerato en Ciencias de la Salud, con mediano interés por la ciencia).

Por su parte, quienes declaran fijarse globalmente en todas las cuestiones planteadas en el enunciado de la pregunta, lo hacen desde un macrointerés que es lo ético: 
"....lo que a mí me interesa y lo que quiero ver en el mundo, que es la humanidad y sobre todo la dignidad, en todas las cuestiones, sociales y culturales..." (Mujer, 32 años, actriz de teatro, Bachillerato de Letras, bajo interés declarado por la ciencia).

Pregunta No 4: Supongamos el siguiente caso ficticio: "una empresa ha instalado un laboratorio de productos químicos al lado de la escuela en que podrían estar matriculados tus hijos. Los vapores que se desprenden pueden ser perjudiciales” ¿Qué actitud adoptarías?

En este caso la totalidad de los participantes declara adoptar una actitud activa que se encontraría entre la movilización (protesta) y la demanda de información. Sirvan de ejemplos estos comentarios:

"Ahí si me movilizaría y podría incluso tomar muchos datos e informarme bien para poder promover una
plata...bueno una actitud de pa..., o hablaría con los padres..." (Mujer, 48 años, actriz de teatro, Grado Medio
de Turismo, bajo interés declarado por la ciencia).
"Protesto, posiblemente no lleve a nada... Yo iría por lo legal, con firmas y abogados..." (Varón, 43 años,
Enseñanza Obligatoria completa, Técnico Forestal, interés medio declarado por la ciencia).
“...si realmente tengo una base firme sobre la que argumentar que esa planta es nociva, pues, intentaría
quejarme, intentaría que alguien hiciese algo, ya sea el Ayuntamiento, recogida de firmas o manifestaciones..."
(Varón, 20 años, Bachillerato Científico, estudiante de la Licenciatura de Filosofía, alto interés por la ciencia
declarado).

Pregunta N 5: Hay personas que están siendo preparadas para viajar e instalarse en Marte en 2030. ¿Qué opinas al respecto?

Se advierte que la mayoría de los encuestados responden aprobando dicho viaje, si bien argumentando a través de intereses individuales.

“...si el hombre es capaz de... Porque a mí me gusta mucho viajar y conocer, entonces si bueno... si hay otro sitio más que pueda ser, por qué no... En principio no me parece una idea.., me gusta, porque me gusta la astronomía entonces me gustaría conocer los planetas..." (Mujer, 32 años, actriz de teatro, Bachillerato de Letras, con bajo interés declarado por la ciencia).

Por su parte, quienes responden alejándose de sus intereses individuales cercanos, mencionan la importancia del conocimiento o una visión de futuro en la que la Tierra no podrá albergar a la gente.

“...yo creo que es que la Tierra está condenada a desaparecer, aunque no la estuviéramos haciendo desaparecer nosotros, da igual, la Tierra va a desaparecer, en el momento que el Sol sea una supernova se acabó..." (Varón, 43 años, director de teatro, Bachillerato en Letras, Licenciado en Geografía e Historia, interés medio declarado por la ciencia).

En contraposición, los que se muestran desfavorables ante la iniciativa de viajar a Marte, argumentan que se deben priorizar las propuestas referidas a nuestro planeta. Así se desprende de esta valoración:

“...Me parece que hay cosas más importantes que viajar a Marte, mejor cuida tu planeta y no vayas a destruir otro..." (Varón, 23 años, Bachillerato Científico, estudiante de la Licenciatura de Filosofía, con interés moderado por la ciencia declarado). 
Pregunta $\mathbf{N}^{\circ}$ 6: Para este viaje deberán resolverse una serie de interrogantes y retos. ¿Te parece que tendremos que esperar al enunciado de nuevas teorías científicas para resolver esos problemas?

En términos generales, la mayoría de los participantes considera que la ciencia es un cuerpo de conocimientos acabado y estático, con el que se podrán resolver todos los problemas a los que nos enfrentemos. Como refleja el siguiente comentario, los avances que puedan acontecer se asocian con aplicaciones o mejoras tecnológicas.

“...aplicando algunas teorías de las que tenemos ahora, de manera digamos, extraña o diferente, haciendo ahí algún cambio, igual funciona alguna pero...Yo creo que tiene que haber avances en lo tecnológico..." (Varón, 20 años, Bachillerato Científico, estudiante de la Licenciatura de Filosofía, con alto interés por la ciencia declarado).

Por su especial interés y significado se alude a la valoración de una participante sobre la confianza en la ciencia. Señala que la forma de trabajo de los científicos cuenta con la refutación como parte de su proceder. En este sentido, las teorías con las que se cuenta hoy ya habrían sido probadas debiendo por ello, confiar en ellas. Descarta que una nueva forma de comprender un problema o el surgimiento de nuevos hallazgos fruto de los avances tecnológicos podría destronar las teorías existentes.

Pregunta No 7: Si tuvieses que asociar la idea de energía que tienes con el suministro eléctrico en una ciudad o con la idea de Reiki (energía positiva)... ¿Por cuál de las dos opciones te inclinarías preferentemente?

Los hallazgos apuntan que tres de los participantes poseen una inclinación total hacia las pseudociencias, cinco no muestran inclinación alguna hacia estas, y el resto lo hacen de manera moderada (ver comentario).

"Yo era absolutamente escéptico y he notado cosas... Entonces como he notado cosas, no solamente en el campo del Reiki sino en otras, insisto, hasta en lo..., hasta en lo..., no ya en el Reiki, en lo paranormal te voy a decir..." (Varón, 43 años, director de teatro, Bachillerato en Letras, Licenciado en Geografía e Historia, interés medio declarado por la ciencia).

"Obviamente es en el suministro electrico, es energía literal como lo entendemos. Lo del Reiki es energía entre comillas, que no, bueno, yo entiendo que es una mentira" (Mujer, 21 años, Bachillerato Científico, estudiante de la Licenciatura de Filosofía, interés medio declarado por la ciencia).

\subsection{Bloque 2: Dimensión Cognitiva}

El bloque II de preguntas gira en torno a la información que aparece en un documento publicitario de venta de cáscara de almendras como biocombustible, destacando sus ventajas frente a otros materiales, cuestión que se visualiza mediante un gráfico. Este texto se entrega 
impreso a los participantes. El objetivo de esta actividad radica en determinar si los participantes reconocen las propiedades de los materiales, si utilizan la gráfica para hacer comparaciones y si son capaces de diseñar un método experimental para comprobar qué material genera más calor por kilogramo.

Se encuentra que casi todos comprenden la contrastación que intenta presentar la publicidad entre distintos materiales, pero respecto al uso de las gráficas para solventar el análisis textual que realiza quien intenta vender la cáscara de almendras, casi la totalidad de los participantes ha corroborado o bien no consultarlas directamente o no revisarlas por entender que se trata e información duplicada. Así lo refleja este comentario:

“...al fin y al cabo la gráfica que te están poniendo aquí es exactamente lo mismo que si coges y lees...” (Varón,
20 años, Bachillerato científico, estudiante de licenciatura de filosofía, alto interés por la ciencia declarado).

Por otro lado, y en referencia al diseño experimental propuesto para comprobar qué material genera más calor, las respuestas obtenidas se distribuyen en dos polos claramente diferenciados: 1) diseños de corte científico: contemplaba la elección de materiales, instrumentos de medida, control de variables, predicción de resultados y manejo de modelos muy ligados a la medición; y 2) diseños de corte vulgar: aludiendo a su experiencia personal y a percepciones sensoriales, asociando más calor producido con intensidad de llamas, tiempo de combustión, volumen de gases, calor detectado a través del tacto o temperatura percibida mediante la piel. Como ejemplos de estos hallazgos se incluyen a continuación dos propuestas, la primera de diseño científico y la segunda vulgar.

“...si no tuviese aparatos suficientes yo creo que una prueba buena sería algo así como, encerrarme en una habitación con una estufa, medir la temperatura inicial, mantener esa temperatura inicial constante en las tres pruebas que haga y en las tres quemar la misma cantidad y ver a cuánto ha subido la temperatura, entonces la que más suba será la que más rendimiento da..." (Varón, 20 años, Bachillerato Científico, estudiante de la Licenciatura de Filosofía, alto interés por la ciencia declarado).

".....habrá que probar.....quemaría... Vería los grados que da.....el aguante que daría, los gases que daría..." (Varón, 43 años, Enseñanza Obligatoria completa, Técnico Forestal, Interés medio declarado por la ciencia).

A continuación, y a modo de resumen de los resultados, se describen los perfiles de cada grupo de sujetos respecto al análisis de las respuestas realizado en el epígrafe previo, procurando con ello determinar algunos patrones comunes que permitan corroborar la validez del instrumento utilizado para evaluar ciertos aspectos referentes a la cultura científica de una selección de ciudadanos.

\section{a) Grupo 1: Los actores}

$>$ Aluden a la metodología del profesorado, tanto para la evocación de emociones positivas como negativas. 
$>$ Su actitud hacia el aprendizaje de las ciencias se encuentra muy relacionada con la forma en que se les enseña.

$>$ Constituyen el grupo que más expresa su pesadumbre respecto a no haber sido exitosos en el aprendizaje de esta área de conocimiento.

$>\quad$ Ninguno de ellos se puede categorizar como participativo en sociedad, sobre todo por el descreimiento referido a que su acción sirva realmente. No obstante demandan la atención en asuntos sociales por parte de los gobiernos.

$>$ Se muestran más beligerantes cuando el asunto es cercano y los puede afectar directamente a ellos a o su gente.

$>$ Desarrollan una visión de la ciencia como cuerpo de conocimientos acabado y de los científicos como genios.

$>$ Su adhesión a las pseudociencias es la más alta comparativamente con los grupos restantes.

$>$ Comprenden íntegramente el texto publicitario sin recurrir a la consulta o lectura de las gráficas. Ninguno plantea un diseño experimental claramente científico ni recurre a modelos.

\section{b) Grupo 2: Alumnado de Filosofía}

$>$ Manifiestan un recuerdo más amable de sus aprendizajes pasados en ciencias, subrayando el hecho de haber dudado incluso sobre si seguir una cerrera científica. También son quienes más insisten en el esfuerzo y trabajo intenso que se les exigía para aprender.

$>$ Es el único grupo que posee estrategias metacognitivas, reconociendo claramente cómo controlar sus procesos cognitivos.

> Poseen la máxima independencia de la metodología del profesor para definir su postura positiva ante el aprendizaje de las ciencias, mencionando características de ellas que los conducen a esta posición favorable.

$>$ Es también en este grupo únicamente donde aparecen integrantes participativos y ante el caso controversial tecnocientífico planteado se muestran más moderados y dialogantes, esperando a contar con información antes de tomar una postura de protesta.

$>$ Poseen nula adhesión a las pseudociencias.

$>$ Comprenden el texto publicitario y la totalidad propone estrategias experimentales científicas para corroborar cuál es el mejor material a seleccionar.

\section{c) Grupo 3: Profesionales forestales}

$>$ Los trabajadores forestales son los menos afectados emocionalmente a favor o en contra respecto a sus éxitos o fracasos en esta área del conocimiento, mostrado una situación igualada con los otros campos disciplinares.

$>$ Son muy poco participativos, sobre todo de forma directa, apelando a las instituciones para que resuelvan los problemas, aún los cercanos que les afecta muy directamente. La postura es que no les corresponde intervenir.

$>$ Comparten una imagen de ciencia como cuerpo de conocimientos acabado con los demás grupos.

No adhieren totalmente a las pseudociencias.

$>$ Comprenden la idea del texto publicitario y todos plantean un diseño experimental pero claramente de tipo vulgar, atados a lo perceptivo (lo que ven, oyen, sienten).

$>$ Adhieren a las pseudociencias pero con ideas confusas.

Respecto a los aspectos emocionales, como se desprende de las líneas previas, quienes muestran marcadas emociones respecto a sus inicios en ciencias vinculan a aquéllas con la metodología del profesor y al mismo tiempo muestran un sentimiento de pesadumbre por no haber podido continuar con estudios en esa área. En el extremo opuesto se encuentran quienes no han tenido gran compromiso con el estudio o la cultura en general y su vinculación emocional con la enseñanza de las ciencias en sus inicios, no es distinta a la que poseen respecto a otras áreas de conocimiento. Sin embargo, un tercer grupo, que habiendo optado por una profesión sin vinculación con la ciencia, poseen una actitud positiva hacia la ciencia, recordando con agrado sus primeros pasos en su aprendizaje. Este grupo cursó Bachillerato 
científico y, al contrario que el primero, es el más independiente emocionalmente respecto a la metodología docente.

En cuanto a su nivel de participación en sociedad, se destaca que no se muestran participativos en general; en el área científica, algunos grupos, solamente cuando el problema es cercano y los afecta personalmente pueden llegar a movilizarse, pero de maneras diferentes: algunos optan por la protesta, otros, los que cursaron el Bachillerato científico, se muestran más prudentes y plantean la búsqueda de información como prerrequisito a la movilización.

En referencia a la unánime visión de ciencia de los participantes, esta responde a una idea de cuerpo de conocimientos acabado y estático, contrapuesto a posturas epistemológicas actuales que reconocen la subjetividad compartida pero cambiante de los científicos (intersubjetividad) como parte del quehacer científico, y en consecuencia, la transitoriedad de las leyes y teorías como característica inherente de la ciencia. Ni aún en los que han cursado Bachillerato científico y por tanto han estado expuestos mucho más tiempo al aprendizaje de las ciencias, se encuentra algún atisbo de una postura epistemológica actual. En consonancia con esta imagen distorsionada de la ciencia aparece la adhesión a las pseudociencias en forma dispar pero con una defensa más fuerte de este pseudoconocimiento por parte del primer grupo.

En relación a sus habilidades cognitivas, sobre todo al uso del pensamiento hipotético deductivo puesto de manifiesto a través de la propuesta de diseño experimental solicitada, es notoria la huella de la enseñanza de la ciencia al nivel de Bachillerato. Únicamente quienes pasaron por ese nivel pudieron hacer una propuesta científica y utilizar modelos.

\section{CONCLUSIONES}

Una de las principales conclusiones de este trabajo radica en el hecho de que quienes logran independizarse emocionalmente de la metodología del profesor de turno y encuentran la motivación y el estímulo en los conocimientos en sí, poseen un nivel de éxito mayor en sus aprendizajes y además mantienen una actitud positiva más allá de si esa área de conocimientos se vincule a su carrera o profesión. Este aspecto, tal como era de esperar por el tipo de instrumento utilizado, los cuestionarios, no ha podido ser detectado por la FECYT. Este hecho es, sin embargo, crucial y tiene repercusiones pedagógicas al nivel de la educación formal, sobre todo en tramos obligatorios, donde los alumnos adquieren los 
elementos que los acercarán o alejarán de un ámbito de conocimiento concreto. Además de utilizar una metodología activa y significativa como reclaman los participantes, los profesores de ciencias deberían ayudar a desvelar lo prodigioso de este cuerpo de conocimientos despertando el placer por la rigurosidad, la predictibilidad, la coherencia, la posibilidad de matematizar, etc. Disfrutar de estas características, más allá de su didáctica sería un requisito de un aprendizaje exitoso que permitiría que los alumnos, tanto los que se dedicarán a profesiones científicas como los que no, posean una actitud positiva hacia la ciencia.

En cuanto a la participación en sociedad y la tendencia a tomar partido solo si la afección es personal, esto puede ser coherente con culturas individualistas como la Europea, aspecto ya contrastado en los resultados de las encuestas de FECYT. También en este tópico la escuela podría implementar acciones que trascendieran las declaraciones realizadas en los currículos donde se insiste en el trabajo en grupo y colaborativo, el bien común, etc. y en todo caso revisar cómo esas intenciones de promoción de lo colectivo se pueden mejorar.

Así mismo, acerca de la visión de ciencia de los participantes y de sus dificultades en el manejo del pensamiento formal, es muy probable que estos dos resultados se deban a la forma en que la ciencia es presentada tanto en la enseñanza formal como en espacios de divulgación y en ese sentido también la escuela debería adoptar una posición. Respecto al primer punto, este se ha encontrado en encuestas de la FECYT, pero el segundo no ha sido explorado.

En suma, el diseño presentado es coherente con las estrategias cuantitativas utilizadas mayormente hasta el momento, aportando de manera complementaria nuevos tópicos que podrían utilizarse en los cuestionarios tradicionales.

Además, aporta la exploración de lo emocional que no es visible desde perspectivas cuantitativas y que enriquece la explicación y comprensión de los significados conferidos a la ciencia. 


\section{Referencias}

Cadena-Iñiguez, P., Rendón-Medel, R., Aguilar-Ávila, J., Salinas-Cruz, E., Cruz-Morales, F., Del Rosario, F., \& Sangerman-Jarquín, D. M. (2017). Métodos cuantitativos, métodos cualitativos o su combinación en la investigación: un acercamiento en las ciencias sociales. Revista Mexicana de Ciencias Agrícolas, 8(7), 16031617.

Costanzo, G., \& Golombek, D. (2020). The quest for scientific culture. Journal of Science Communication, 19(1), R01. doi: https://doi.org/10.22323/2.19010601

Emans, B. (2019). Interviewing: Theory, techniques and training. Oxford: Routledge

Espín, J. V. (2002). El Análisis de contenido: una técnica para explorar y sistematizar información. XXX. Revista de Educación, 4, 95-106.

Fundación Española para la Ciencia y la Tecnología, FECYT (2017). Percepción social de la ciencia y la tecnología. Madrid: Editorial MIC.

Jentoft, N., \& Olsen, T. S. (2019). Against the flow in data collection: How data triangulation combined with a 'slow' interview technique enriches data. Qualitative Social Work, 18(2), 179-193. doi: https://doi.org/10.1177/1473325017712581

Mena, H. C., Troyano, A., \& Aguilera, X. (2020). Alfabetización funcional: más que un aprendizaje, una experiencia de vida. Praxis educativa, 24(1), 1-9. doi: https://dx.doi.org/10.19137/praxiseducativa-2020-240111

Morgan, D. L. (2019). Commentary after triangulation, what next? Journal of Mixed Methods Research, 13(1), 611. doi: https://doi.org/10.1177/1558689818780596

Olaz, A. (2008). La entrevista en profundidad: justificación metodológica y guía de actuación práctica. Madrid: Septem.

Osorio, C. (2018). Controversias en la historia de la ciencia y cultura científica. Revista Iberoamericana de Ciencia, Tecnología y Sociedad, 13(37), 297-299.

Strasser, B. J., Baudry, J., Mahr, D., Sanchez, G., \& Tancoigne, E. (2018). Citizen Science? Rethinking science and public participation. Science and Technology Studies, 32(2), 52-76. 